\title{
La sociología española y el antisemitismo Entre prejuicios pasados y clave civilizatoria
}

\author{
Benno HERZOG \\ Universitat de València \\ benno.herzog@uv.es
}

Recibido: 10-12-2013

Aceptado: 13-10-2014

\section{Resumen:}

Se puede detectar una notable ausencia de estudios sociológicos en España sobre el antisemitismo, en cambio sí se detectan tendencias antisemitas preocupantes y estereotipos antijudíos por encima de la media europea.

El presente artículo pretende ofrecer explicaciones sobre esta ausencia.

Para ello se muestran los problemas estructurales que ha tenido la sociología, ya desde sus principios, para captar el fenómeno del antisemitismo. Además se ofrecen explicaciones relacionadas con la situación histórico-social específica de España, en general, y con la sociología Española, en concreto.

Se destaca que por su carácter racionalista y a excepción de unos pocos autores -considerados marginalizados por la investigación diaria- la sociología ha mostrado la existencia de grandes problemas para captar el fenómeno del antisemitismo. La situación histórica específica, el franquismo, la disputa por la memoria histórica y la tardía institucionalización de la sociología, podrían igualmente explicar la falta de un interés sociológico por el tema.

El presente artículo muestra que el estudio del antisemitismo, no sólo resulta relevante para progresar hacia la desaparición de este lastre en todas sus vertientes, sino que también, la reflexión sobre el antisemitismo puede ayudar además a la sociología a reconocer sus propios problemas epistemológicos. Puede servir para criticar y mejorar los instrumentos de investigación sociológicos, mostrar los límites del enfoque sociológico y poner al descubierto la importancia del trabajo interdisciplinar para el entendimiento de ciertos fenómenos sociales. En este sentido, el antisemitismo, lejos de ser un tema marginal, podría ser considerado tema clave en el proceso civilizador y para ayudarnos a descifrar la sociedad actual.

Palabras clave: Antisemitismo; antijudaísmo; antisionismo; España; Israel; sociología; ilustración.

\section{Spanish Sociology and Anti-Semitism Between passed prejudice and key to civilization}

\begin{abstract}
:
There is a significant lack of sociological research in Spain about anti-Semitism. At the same time there are alarming anti-Semitic tendencies and anti-Jewish stereotypes which are above the European average.

This article aims to explain this lack of sociological research about anti-Semitism in Spain.

Therefore two types of explications are offered: on the one hand side some structural problems will be shown which sociology in general had since its beginnings and which complicate the understanding of anti-Semitism. Furthermore explications regarding the specific social and historic situation in Spain and of Spanish sociology in particular will be exposed.
\end{abstract}


It will be shown that for its rationalistic character and with the exception of very few authors -who are considered marginalized for practical research- sociology in general has had enormous problems in understanding anti-Semitism. The specific historic situation, Francoism, the dispute about the historic memory and the delayed institutionalisation of sociology could also explain the lack of sociological interest in the topic especially in Spain.

The article shows that the study of anti-Semitism is not only relevant for struggling against this burden of society in many of its variants. Furthermore, thinking about anti-Semitism can help sociology to recognise its own epistemological problems. It can serve to criticise and improve instruments of sociological research by showing the limitations of the sociological approach and to uncover the importance of interdisciplinary research for understanding specific social phenomena. In that sense, anti-Semitism, far from being a marginal subject, can be considered a key topic in the process of civilisation and it can help us to decipher the contemporary Spanish society.

Keywords: Anti-Semitism; anti-Judaism; anti-Zionism; Spain; Israel; sociology; Illustration.

\section{Referencia normalizada}

Herzog, B. (2014): "La sociología española y el antisemitismo. Entre prejuicios pasados y clave civilizatoria”, Política y Sociedad, 51 (3), pp. 817-839.

Sumario: Introducción. 1. Cinco formas de antisemitismo. 2. Estructura común. 3. La sociología y el antisemitisme. 4. España y la sociología Española. 5. Conclusiones. Bibliografía.

\section{Introducción}

Como ciencia de la sociedad, la sociología, con sus teorías y sus investigaciones empíricas, depende del mundo social en el que se ubica. Las teorías sociales suelen necesitar para su surgimiento y su difusión de tradiciones teóricas, de disposiciones cognitivas y una realidad social que las haga entendibles para un sector amplio de la sociedad y con el que retroalimentarse. De esta forma, la Teoría de la Acción Comunicativa (Habermas, 1981) no sería posible sin los movimientos alternativos, estudiantiles, feministas, ecologistas, etc., de los años 80 , y tampoco hubiera tenido la Teoría de la Sociedad del Riesgo (Beck, 2001) su éxito sin la evidencia del accidente de Chernóbil que tuvo lugar en el mismo año que la publicación de la primera edición del libro. Las investigaciones empíricas, a su vez, dependen en gran medida de las percepciones que se tengan de los problemas sociales, de modas o -reflejo de ambos-, de fondos de investigación. De este modo, se ha podido mostrar, por ejemplo, cómo el aumento de las investigaciones sobre "exclusión social" está relacionado con el surgimiento de fondos económicos de investigación para este tema y el retroceso de otros conceptos que describen injusticias sociales, tales como "la explotación" o "la alienación" (Herzog, 2011).

La relación entre el contexto social y teorías o investigaciones sociológicas, supone el marco de este artículo sobre la falta de una sociología del antisemitismo moderno en España. Si buscamos en la base de datos internacionales ISI Web of Knowledge el término "antisemitism" en los últimos 20 años, encontramos 308 entradas con autores de EEUU, 116 con autores británicos, 71 con autores alemanes y sólo 8 entradas con autores españoles. Si analizamos con más detenimiento estas 
8 entradas, veremos que 6 de ellas no se dedican al antisemitismo moderno, sino a fenómenos históricos. Quedan sólo dos textos: por un lado un artículo del filósofo José Antonio Zamora (2012), que tiene como objetivo no el antisemitismo sino el racismo y la xenofobia, al preguntarse si la Teoría Crítica del antisemitismo puede ayudar a entender estos fenómenos. Por otro lado, encontramos un artículo genuinamente sociológico con la coautoría de la investigadora de Madrid y Salamanca Paula López y con la primera autoría de Alejandro Baer (Baer y López, 2012), en aquel momento investigador en Alemania y ahora director del Centro de Estudios de Holocausto y Genocidio en la Universidad de Minnesota. Baer defendió en 2003 su tesis sobre la memoria del holocausto en la Universidad Complutense de Madrid. También las bases de datos españolas muestran una imagen parecida. La base de datos de ciencias sociales ISOC del CSIC encuentra un total de 289 documentos sobre antisemitismo, en su inmensa mayoría estudios históricos. Limitando la búsqueda al campo de la sociología y las ciencias políticas quedan sólo 29 documentos. De nuevo la mayoría son estudios históricos, mezclan el antisemitismo con otras formas de discriminación o están escritos por autores extranjeros. Y como último indicio de la ausencia de la investigación del antisemitismo en la sociología española podemos constatar que en España sólo existe un único equipo de investigación dedicado al análisis del antisemitismo, pero de nuevo, desde una perspectiva filosófica y no sociológica.

El objetivo del presente estudio es desarrollar explicaciones sobre la ausencia de investigaciones sobre antisemitismo en la sociología española. Algunas de estas explicaciones se centran más en la dificultad de la sociología en general para captar el fenómeno del antisemitismo, mientras que otras ponen en evidencia la falta de consciencia sobre antisemitismo en España, y finalmente, otras explicaciones combinan ambos enfoques.

De antemano sólo quiero excluir dos explicaciones para la ausencia de investigaciones sobre antisemitismo en España. La primera, expresada de forma prominente por el entonces presidente del gobierno español Rodríguez Zapatero: "No hay antisemitismo en España, en ninguna de sus expresiones. [...] hoy en día no hay actos antisemitas en España. Antisemitismo era la dictadura de Franco" (cit. según Emergui, 2009). Los estudios realizados por la Anti-Defamation League (ADL) muestran lo contrario. En casi todos los enunciados antijudíos, la población española destaca por encima de la media de Europa occidental (Anti-Defamation League, 2014). Respecto a los actos de los que Zapatero decía eran ausentes, hay que decir que cada año el informe del observatorio de antisemitismo en España contempla una gran cantidad de actos antisemitas, tanto legales como delictivos, en territorio español (p.ej. Observatorio de Antisemitismo en España, 2013).

1 Se trata del grupo "Filosofía después del Holocausto" de CSIC con Reyes Mate como investigador más destacado. 
La otra tesis que quiero excluir de antemano es la irrelevancia del antisemitismo para la sociología moderna después de la segunda guerra mundial. Demasiados autores importantes y ampliamente recurridos en la sociología contemporánea española (p.ej. Adorno 1998; Zygmunt Bauman, 1989; Horkheimer y Adorno, 2010; Talcot Parsons, 1942; Moishe Postone, 1986; o, aunque no es socióloga, Hannah Arendt, 2006), han elaborado teorías sobre el antisemitismo y la importancia del Holocausto en la sociedad contemporánea. Bauman describe su propia experiencia con la investigación del Holocausto: "El Holocausto no era un cuadro en la pared, sino una ventana mediante la cual se visibilizaron cosas que normalmente pasaban inadvertidas. Y lo que surgió no sólo concierne a los autores, víctimas y testigos del crimen, sino que posee la más alta relevancia para todos los que viven hoy en día y quieren vivir también en el futuro" (Bauman, 1989: vii). Para Horkheimer y Adorno (2010a) el antisemitismo burgués "tiene una causa específicamente económica: el disfraz del dominio en la producción" (182). Y para Salzborn (2010) el antisemitismo es directamente el "modelo negativo de la modernidad". Sin juzgar estas afirmaciones podemos decir que hay suficientes indicios importantes como para analizar el antisemitismo moderno y el Holocausto, no sólo bajo el aspecto de un asunto meramente judío, sino también vinculándolo con la modernidad, el proceso de civilización y el modo de producción capitalista. Si el antisemitismo y el Holocausto realmente no son resultado del fracaso de la Ilustración y de la modernidad sino un producto de las mismas, entonces tendríamos importantes razones para dedicarnos a su estudio, también desde la sociología española.

Con el fin de delimitar el objeto del presente artículo, el antisemitismo, presentaré en un primer paso las cinco formas básicas de antisemitismo, tal como se discuten actualmente en la literatura científica. En la segunda parte abordaré las diferencias y similitudes que justifican la unión de varios fenómenos bajo un único término, "antisemitismo". En una tercera parte desarrollaré las primeras explicaciones para la ausencia de estudios sobre antisemitismo, mostrando los problemas estructurales que ha tenido la sociología desde sus comienzos para captar el antisemitismo. Y finalmente, en la cuarta parte de mi artículo, abordaré explicaciones que tienen que ver con la situación histórico-social específica de España, en general, y con la sociología Española, en concreto.

\section{Cinco formas de antisemitismo}

Definir lo que es antisemitismo resulta tarea espinosa y controvertida. No existe una única definición clara y ampliamente aceptada. La definición más común es la declarada como definición de trabajo del Observatorio Europeo del Racismo y la Xenofobia (EUMC), que ahora es la European Union Agency for Fundamental Rights (FRA). Esta definición es utilizada también por otras organizaciones como el European Forum on Antisemitism o, en España, el Observatorio del Antisemitismo en España. Esta definición abarca una página entera, contiene expresiones conscien- 
temente indefinidas e incluye una serie poco ordenada de ejemplos. La definición comienza así: "Antisemitismo es una determinada percepción sobre los judíos que puede expresarse como odio hacia su persona. Las manifestaciones verbales y físicas del antisemitismo se dirigen tanto contra personas judías o no judías como contra sus bienes, instituciones comunitarias o lugares de culto. Estas manifestaciones también pueden tener como objeto al Estado de Israel, concebido como una entidad colectiva judía." (European Forum on Antisemitism, 2005).

Si nos preguntamos en qué exactamente consiste esta "determinada percepción" o estas "manifestaciones verbales y físicas" la definición no nos resulta de mucha ayuda ya que sólo menciona algunos ejemplos tales como negar el Holocausto o comparar a la política israelí actual con la de los nazis. La confusión terminológica se vuelve aún más grande si tenemos en cuenta que algunos autores como Taguieff proponen el término genérico judeofobia, bajo el cual el antisemitismo sólo sería "el odio a los judíos ideológicamente organizado" (Taguieff, 2010: 125). No obstante, en la sociología internacional se suele preferir el término antisemitismo, como se puede ver por ejemplo en la red de investigación sobre "relaciones étnicas, racismo y antisemitismo" de la asociación europea de sociología (ESA) y en el historial de las ponencias en sus congresos.

Bajo el término antisemitismo encontramos cinco tipos ideales, aunque las denominaciones en la práctica no son unívocas. Son estos el antijudaísmo, el antisemitismo racial/nacionalista, el antisemitismo secundario, el antisemitismo islámico y el antisionismo antisemita o antisemitismo antisionista. La literatura conoce además el antisemitismo estructural (véase p.ej. Zamora y Maiso, 2012) que, como se explicará más adelante, aunque lleva el nombre de antisemitismo, no debe ser considerado antisemitismo per se, puesto que no se dirige contra los judíos (sea como religión, "raza", o ciudadanos de Israel) sino que es más bien una estructura de pensamiento afín al antisemitismo.

El antijudaísmo, también nombrado antisemitismo tradicional o antijudaísmo cristiano, es la discriminación de los judíos basada originalmente en argumentos religiosos, pero que se han convertido a lo largo de los siglos en "conocimiento" popular muy arraigado en la cultura cristiana. La negación del judaísmo a reconocer a Jesús Cristo como el Mesías y la culpabilización de los judíos en general por la muerte de Jesús, son quizá los argumentos más básicos y recurrentes en esta forma de antisemitismo. A partir de ahí se ha creado una gran serie de calumnias sobre los judíos: rituales de asesinatos de niños cristianos, blasfemias, profanación de hostias consagradas o la responsabilidad por determinadas enfermedades y epidemias, son quizá las leyendas más conocidas. En este contexto también hay que mencionar los estereotipos que califican a los judíos como usureros, avariciosos, maestros del engaño y vengativos. Son estos últimos los estereotipos que, de forma más intacta, han pasado por la Ilustración y se encuentran hoy en día también como parte de otras formas de antisemitismo.

Frente a esta forma de antisemitismo tradicional, todas las demás formas suelen considerarse antisemitismo moderno. El propio término de antisemitismo fue una creación del siglo XIX con la que se pretendía distinguirse del odio a los judíos, 
considerado retrógrado y emocional. Frente al viejo antijudaísmo surgió, con pretensiones científicas, el antisemitismo al que se referían con orgullo gran parte de los nacionalistas europeos a finales del siglo XIX y principios del siglo XX. En la literatura se conoce este tipo de antisemitismo como antisemitismo racista, racial o nacionalista (véase p.ej. Holz, 2001 y Salzborn, 2013). Presenta el intento de utilizar las nuevas teorías sobre razas y caracteres nacionales consideradas como científicas para justificar la discriminación de los judíos. A estos ya no se les consideraba un grupo religioso, sino una raza. La noción de raza se mezclaba con el nacionalismo y la creación de los Estados naciones en el siglo XIX. En esta idea, según la cual los pueblos se organizan en Estados nacionales, los judíos no sólo aparecían como otro pueblo u otra raza, sino como la anti-raza por antonomasia. Su mera existencia ponía en duda el principio organizador que tenían en mente los nacionalistas europeos (Holz, 2001). Los judíos se convertían así en lo contrario de la nación. Como pueblo sin Estado representaron la no-identidad frente a las identidades nacionales europeas. Frente a la comunidad (Gemeinschaft) de la patria ellos presentaron la organización abstracta, la sociedad (Gesellschaft) y la falta de cultura propia. Gran parte de lo que se temía y que no se entendía en el proceso de la modernización se achacaba a los judíos. Se identificó a los judíos con el dinero y los intereses, con el poder organizado globalmente y en secreto, y tanto con el capitalismo como con el bolchevismo, ambos entendidos como conspiraciones.

Aquí ya se puede apreciar que el antisemitismo moderno es más que un prejuicio parcial y ocasional. El antisemitismo es un modo de explicación, una semántica, una cosmovisión (Holz, 2001). Ayuda a entender el mundo, su organización política, económica y social. Como muestra el ejemplo de la responsabilización de los judíos, tanto por el capitalismo como por el bolchevismo, o las teorías de la conspiración, las cuales se presentan como inmunes a irritaciones y contradicciones: afirmaciones opuestas parecen encontrar fácilmente su lugar en una misma semántica. El antisemitismo se convierte en ideología o cosmovisión (Claussen, 2012), en estructura psicológica o en resentimiento (véase Salzborn, 2013) y escapa de esta forma a la argumentación racional.

El imaginario de los judíos como raza incluye la incapacidad lógica de cambiar la clasificación designada. Ni el renunciar a su religión, ni el declararse patriota ferviente, podrían despojar de su estatus a los que una vez hayan sido identificados como judíos. Lo que es más, en la lógica del antisemitismo racista/nacional, todos los intentos de asimilación tenían que ser considerados actos sospechosos de infiltración. Si los judíos representan la anti-raza y, con ello, lo contrario a la nación, entonces también la idea de judío alemán o judío francés se tenía que convertir en contradicción. La existencia de la diversidad chocaba con la pretensión homogeneizadora, tanto del universalismo de la Ilustración como del nacionalismo de los Estados europeos.

Después de la Segunda Guerra Mundial y la barbarie del Holocausto, el antisemitismo ya no era ninguna referencia positiva. Aunque con ello el antisemitismo racista/nacional no desapareció del paisaje europeo, al menos en el espacio público hegemónico ya no se suele encontrar justificaciones abiertas a este tipo de antisemi- 
tismo. No obstante, el antisemitismo resulta ser muy versátil y poco después de la Segunda Guerra Mundial surge en Alemania, pero también en otras partes del mundo, el antisemitismo secundario, también denominado antisemitismo democrático (Holz, 2005: 54ss), denominado así porque este sí se encuentra en el espacio público democrático. Este antisemitismo, después de Auschwitz, que no se justifica a pesar del Holocausto sino por razón de éste, ha sido resumido por el psicoanalista judío Zvi Rex en una formulación brillante: "Los alemanes no perdonarán nunca Auschwitz a los judíos." (Zvi Rex, cit. según Broder, 1986: 125). Como reversión de culpa, esta forma de antisemitismo es capaz de disculpar por un lado a los agresores y culpabilizar al mismo tiempo a las víctimas. Algunas formas extendidas de disculpar lo hecho consisten en insistir en la ignorancia de gran parte del pueblo alemán sobre el exterminio de los judíos, en enumerar el Holocausto como otra más de las atrocidades cometida antes, después y durante la Segunda Guerra Mundial, o en ensanchar el daño producido contra el propio grupo. Si el Holocausto no fue peor (sino incluso menos malo) que el bombardeo a Dresde, si el Holocausto fue sólo una reacción al y copia del Gulag, ${ }^{2}$ entonces pierde su carácter singular. Algunas formas comunes de culpabilizar a los judíos son suponer algún tipo de poder o amenaza por parte de estos, o insistir en el carácter violento de sus exigencias de reparación. En la combinación de la exculpación y la culpabilización, la dimensión del tiempo hace mover la balanza en contra de los judíos. Mientras "nuestra" culpa es pasada y "nosotros" ya hemos pagado por los hechos, las exigencias económicas o morales de los supervivientes y sus predecesores siguen en la actualidad. Así, en 2014 respondieron con "probablemente cierto" a la afirmación "los judíos aún hablan demasiado sobre lo que les sucedió en el Holocausto", el 52\% de los alemanes, el $52 \%$ de los austriacos y el $48 \%$ de los españoles (Anti Defamation League, 2014). Esta queja se combina con la idea de la organización a escala global de los judíos y su supuesto poder, que les lleva finalmente a la creación de la "industria del Holocausto" (Finkelstein) en la que los judíos explotan la memoria del Holocausto con fines económicos y políticos. Los paralelismos con la imagen del judío sin escrúpulos y avaricioso son más que notables.

El antisemitismo islámico puede ser percibido como el resultado de una exportación del antisemitismo moderno, europeo, a las condiciones específicas del mundo árabe o musulmán. A partir de los años 20 del siglo pasado, el antisemitismo moderno, europeo, se encuentra con un proceso de modernización y descolonización de los países árabes. Para entender la relación entre descolonización y antisemitismo es importante comprender que la adquisición de tierra por organizaciones y colonos judíos era percibida por la mayoría árabe como colonización bajo el protectorado británico. En la primera mitad del siglo pasado, los movimientos anticoloniales tenían así los mismos enemigos que los nacionalsocialistas: Gran Bretaña, el

\footnotetext{
${ }^{2}$ Así por ejemplo en el Historikerstreit alemán (disputa de historiadores - véase Piper, 1987).
} 
bolchevismo y los judíos. No nos ha de sorprender que gran parte del movimiento anticolonial contra Gran Bretaña y Francia se entusiasmara por el nacionalsocialismo. Uno de los primeros antisemitas árabes más destacados de esta época fue el muftí de Jerusalén, Muhammad Amin al-Husayni. Era colaborador estrecho con el régimen nazi y sus ideas influyeron en amplias partes del mundo árabe. Aunque la simpatía abierta con el nacionalsocialismo retrocedió radicalmente después de la Segunda Guerra Mundial, el antisemitismo como semántica, como cosmovisión capaz de explicar la realidad económica, política y social, se mantiene en gran parte del mundo árabe. Así que el conflicto judío-árabe (o israelí-árabe, si así se prefiere) de Oriente Próximo no se percibe mediante una interpretación propia, local, sino como parte de una semántica global que vincula a los judíos/Israel con el colonialismo, con redes globales de poder económico y militar (p.ej. "lobby judío" en EEUU) y se percibe la existencia de Israel no sólo como amenaza para la paz en la región sino incluso para la paz mundial. ${ }^{3}$ No nos ha de sorprender que en la Carta fundacional de Hamás, todavía hoy en vigor, se haga referencia a los "Protocolos de los sabios de Sion", falsificación antisemita rusa de 1902 que habla de una conspiración judía mundial. La Carta, además, identifica a los judíos detrás del poder de los medios de comunicación, de la revolución francesa, del bolchevismo y muchas apariencias más del mundo moderno (para la versión inglesa de la Carta véase MEMRI, 2006). A raíz tanto de las migraciones desde el mundo árabe como de movimientos de solidaridad anticoloniales en el llamado primer mundo, las ideas del antisemitismo islámico se están reimportando a Europa.

El conflicto judío-árabe y su codificación como conflicto colonial es una de las fuentes del antisemitismo antisionista, también a veces denominado antisionismo antisemita (p.ej. Baer, 2012) según si se quiere subrayar que se trata de antisemitismo que aparece en forma de antisionismo o si se trata de antisionismo que utiliza argumentos antisemitas. En esta forma de antisemitismo se fusionan la interpretación del conflicto alrededor de Israel del antisemitismo islámico con el apoyo oficial de la lucha anticolonial y antiimperialista de la Unión Soviética. Mediante movimientos de solidaridad con estas luchas y organizaciones afines a las ideas de la Unión Soviética se extiende el antisionismo a gran parte de la izquierda europea.

Aquí resulta imprescindible hacer un pequeño paréntesis y diferenciar este antisionismo, que se presenta como laico, del antisionismo ultra-ortodoxo. El sionismo como idea y movimiento político moderno comenzó a finales del siglo XIX. Nathan Birnbaum y Theodor Herzl quizás pueden ser vistos como los defensores más influyentes de este movimiento que buscaba la creación (y posteriormente el mantenimiento y la defensa) de un Estado nacional judío. Ya en sus comienzos el sionismo fue una reacción al antisemitismo en Europa. Desde el nacionalsocialismo,

${ }^{3}$ Las encuestas de Eurostat muestran con regularidad que Israel está considerado como el país que más amenaza la paz mundial, por encima de otros países como EEUU, Corea del Norte, Irán, Irak o China. 
como muy tarde, la vida judía en gran parte de Europa resultó imposible. El sionismo encuentra dos respuestas dentro de la propia comunidad judía. Una de ellas, muy común antes de la Segunda Guerra Mundial, era la posibilidad de superar el antisemitismo en Europa. Según esta posición el sionismo y el antisemitismo quieren lo mismo: la exclusión de los judíos de Europa. Los antisionistas, que así argumentaron, apostaron por lo contrario, por la integración, la asimilación y la igualdad de derecho dentro de las sociedades europeas. Otro argumento dentro del judaísmo es el presentado por algunos grupos ultra-ortodoxos, tanto fuera como dentro de Israel. Según estos, la creación de un Estado judío sería una blasfemia. Siguiendo la interpretación de la Tora de estos grupos, sólo Dios puede librar a los judíos de la situación de Diáspora y lo hará enviando al Mesías.

En esta forma de antisionismo hay que distinguir aquello que utiliza argumentos antisemitas. Son aquellos sobre todo las retóricas que hacen referencia al poder, al dinero, conspiraciones, redes globales de los judíos, aunque el término judío suele estar sustituido por el de Israel o sionista ${ }^{4}$. Otros ejemplos de la retórica antisemita dentro de algunas corrientes del antisionismo, consisten en agrandar las injusticias cometidas por Israel o igualar la situación de la población palestina actual con la de los judíos durante en nacionalsocialismo. Parecido al antisemitismo nacional, también estas formas del antisemitismo antisionista, perciben la incapacidad de los judíos de crear su propia nación y se les niega lo que a todas las demás naciones se les concede. Por ello, en la ya mencionada definición de trabajo utilizada por la Unión Europea, se define como antisemitismo respecto al Estado de Israel.

- "Negar al pueblo judío el derecho de autodeterminación, por ejemplo, afirmando que la existencia del Estado de Israel es un proyecto racista.

- Aplicar una doble moral, exigiendo al Estado de Israel un comportamiento que no se espera y demanda de ningún otro Estado democrático.

- Usar símbolos e imágenes asociadas con el antisemitismo clásico (por ejemplo, la acusación de que los judíos asesinaron a Jesús o el libelo de la sangre) para caracterizar a Israel o a los israelíes.

- Realizar comparaciones entre la política israelí actual y la de los nazis.

- Responsabilizar colectivamente a los judíos por las acciones del Estado de Israel." (European Forum on Antisemitism, 2005).

Para no evocar la idea de que toda crítica a la política de Israel sería antisemitismo, se han elaborado algunas ayudas orientativas. Así que Salzborn (2013) cita por ejemplo el test 3-D de Sharansky, según el cual la deslegitimación, demonización y el doble rasero serían criterios para diferenciar una crítica permisible (independientemente de si esta crítica es acertada o no) de una forma de antisemi-

${ }^{4}$ Holz (2005: 88) cita un ejemplo especialmente descarado de una revista de la Unión Soviética de 1972 en que se publicó un texto antisemita de 1906 simplemente cambiando la palabra "judío" por "sionista". 
tismo antisionista. Salzborn ofrece además la diferenciación entre crítica y resentimiento. En la crítica domina la facticidad racional. El crítico es capaz de revisar su propia posición con la ayuda de nuevos hechos. El resentimiento antisemita está vinculado a los afectos y se caracteriza por la incapacidad de cambiar la posición propia. Haga lo que haga Israel, para el antisemita la posición en contra del Estado de Israel está determinada de antemano y se inmuniza de esta manera contra hechos diferenciadores.

De estas cinco formas básicas quisiera distinguir una sexta forma de pensamiento que en sí aún no presenta ninguna forma de antisemitismo porque no va dirigida contra los judíos, ya sea como religión, raza o ciudadanos de Israel. Estoy hablando de lo que comúnmente se conoce como antisemitismo estructural ${ }^{5}$. Se trata de una forma de pensar que "no se refiere inmediatamente a los judíos, sino a fenómenos que el antisemitismo primario identifica con ellos: por ejemplo el no tener raíces, una intelectualidad excesiva, el secretismo misterioso o la deslealtad hacia los poderes establecidos." (Claussen, 2012: 29). Más allá de estos ejemplos se pueden nombrar también ciertas críticas muy extendidas al dinero, el capital financiero o la especulación. Podríamos decir que se trata de una forma mutilada de crítica al capitalismo que comparten amplios sectores de la población. Ya el nacionalsocialismo conocía la diferencia entre capital usurero y capital creador (industrial), diferenciación muy común en amplias partes de los movimientos sociales, hoy en día, que critican a los bancos defendiendo al mismo tiempo la industria (nacional) como solución a los problemas económico-sociales (véase Zamora y Maiso, 2012). Como decía antes, esta forma del pensamiento en sí aún no puede ser considerada antisemitismo. No obstante, la podemos entender como estructura básica capaz de transportar y comunicar modos específicos de pensamiento entre diferentes grupos sociales y entre distintos tiempos históricos que facilitan especialmente la comunicación de contenidos antisemitas. Se convierte en antisemitismo en cuanto se identifica a estos poderes misteriosos, anteriormente nombrados, con la figura del judío. Por tanto, puede ser considerado precursor o estructura básica del antisemitismo o simplemente una forma primitiva de crítica al capitalismo.

En la presentación de estas $5+1$ formas de antisemitismo ya se han podido vislumbrar algunas disputas sobre denominaciones. Otras diferenciaciones son posibles y se entrecruzan con las categorizaciones aquí presentadas. Ya se ha mencionado la diferenciación tradicional vs. moderna en la que el antisemitismo moderno se presentaría en todas las formas salvo en la primera. Otra posibilidad es la diferenciación del antisemitismo de derechas (p.ej. antijudaísmo, antisemitismo racista/nacional) vs. antisemitismo de izquierdas (p.ej. antisemitismo antisionista, antisemitismo estructural). No obstante, en la realidad raras veces encontramos estos tipos ideales y más bien encontraremos mezclas y alianzas políticas que a primera

5 Claussen (2012: 29) denomina esta forma "antisemitismo secundario", lo cual puede provocar muchas confusiones con la tercera forma de antisemitismo aquí presentada. 
vista podrían parecer extrañas: la izquierda y derecha juntas contra el poder de los bancos (antisemitismo estructural), o movimientos emancipadores con movimientos represivos clericales contra la política de Israel, por sólo nombrar algunos. Finalmente podríamos hacer referencia también a la diferencia entre antisemitismo abierto (las dos primeras formas) vs. antisemitismo latente o no reconocido. El propio carácter versátil del antisemitismo se presta para negar las propias tendencias antisemitas. Así que la alusión al antisemitismo racial de la derecha puede servir a cierta izquierda para negar el antisemitismo en las filas propias.

A pesar de la complejidad del tema, podemos resumir que ciertas formas, aunque no son exclusivas de una región, un tiempo histórico o una ideología política, pueden ser dominantes o al menos más comunes en un contexto específico que en otro. Así, mientras el antijudaísmo fue dominante en un tiempo histórico donde el poder de la Iglesia era mayor (en España quizá hasta los años 70 del siglo pasado), el antisemitismo racial/nacional fue la forma dominante de los Estados europeos en la creación y definición de sus Estados nacionales desde finales del siglo XIX hasta la segunda guerra mundial. Ambas formas sobreviven hoy en día en círculos ultraconservadores o de extrema derecha. En el espacio público encontramos hoy en día de una forma más obvia las otras formas de antisemitismo. Mientras el antisemitismo democrático se encuentra en el centro político de la sociedad, el antisemitismo antisionista es más dominante en la izquierda antiimperialista y el antisemitismo islámico se encuentra sobre todo en países árabes y en comunidades de inmigrantes de estos países en Europa.

\section{Estructura común}

Si aquí se defiende la posición de que todas estas manifestaciones son el resultado de una misma estructura, de un antisemitismo latente, entonces también se debe decir en qué consiste esta estructura antisemita básica. Con Klaus Holz (2001, 2005) podemos diferenciar cuatro formas básicas de argumentar -Holz las llama semánticas- inherente a todo tipo de antisemitismo: 1. La diferenciación entre comunidad y sociedad, 2. Poder y conspiración, 3 . La reversión agresor-víctima y 4. La figura del tercero.

1. Todas las formas del antisemitismo moderno incluyen alguna variante de queja antimoderna: la pérdida de la comunidad, la destrucción de las tradiciones, de formas de convivencia solidaria, armónica y auténtica. Se lamenta la competencia del individualismo y un dominio abstracto. Hasta aquí "sólo" tendríamos elementos del antisemitismo estructural. Pero se convierte en antisemitismo dirigido contra los judíos en cuanto se identifica a estos con las tendencias de pérdida de comunidad "natural". Podemos nombrar algunos ejemplos de esta expresión de identificación de los judíos como antagonista de la comunidad auténtica: en el nacionalsocialismo, los judíos eran descritos como desarraigados; más de la mitad de la población Europea $(65 \%$ de los españoles) afirma que los judíos son más leales a Israel que a su propio país 
(Anti Defamation League 2014); Hamás defiende abiertamente una ideología antimoderna (p.ej. frente a las mujeres) e identifica las tendencias modernas con el sionismo (MEMRI, 2006); muchos comentaristas políticos afirman la incapacidad de los judíos para formar su propio Estado, es decir, se niega que el nacionalismo judío pudiera ser un nacionalismo como cualquier otro.

2. En todas las formas del antisemitismo se supone algún tipo de conspiración vinculada con un enorme poder de los judíos, ya sea la creencia en la veracidad de "Los Sabios de Sion" o la creencia de que los judíos estuvieron tanto detrás de la Revolución Francesca como detrás del bolchevismo y de las guerras mundiales como afirma Hamás (MEMRI, 2006). Las formas más comunes en Europa de suponer un poder judío que se despliega mundialmente en secreto, es la afirmación de que los judíos/Israel está detrás de los medios de comunicación, de Hollywood, de la política exterior de EE.UU. o detrás de bancos y bolsas (Holz, 2005: 27ss).

3. La suposición de un poder enorme de los judíos/Israel/los sionistas forma parte de una tercera semántica, la tergiversación de agresor a víctima. Nunca el antisemita se considera a sí mismo agresor. Más bien afirma la necesidad de defensa contra un poder imaginado como enorme. Para los nacionalsocialistas el exterminio de los judíos se convirtió en cuestión de supervivencia nacional. Los judíos eran percibidos como parásitos o como cáncer en el cuerpo nacional que chupa de su víctima hasta dejarle sin vida. En relación con el antisionismo antisemita, esta tergiversación de la realidad se presenta, por ejemplo, en la ignorancia de que algunas organizaciones palestinas aspiran abiertamente "a la destrucción de Israel, desprecian y ningunean derechos individuales de libertad y derechos humanos y quieren instaurar un sistema totalitario de la umma islámico" (Salzborn, 2013: 13). En vez de ello, el antisionismo antisemita se imagina a Israel como Estado racista que planifica y lleva a cabo un genocidio contra la población palestina. Según esta ideología la lucha contra Israel/el sionismo se convierte en defensa de un pueblo oprimido (los palestinos) contra unos agresores casi omnipotentes. El antisemitismo secundario conoce esta reversión, por ejemplo, en la afirmación de que los judíos exprimen a Alemania mediante la instrumentalización del Holocausto.

4. La figura semántica del tercero es la que quizá más claramente diferencia el antisemitismo de otras formas de discriminación como el racismo. A la ideología antisemita subyace una diferenciación binaria en "nosotros" y "los otros". Independientemente de si el propio grupo está pensado en términos nacionales, raciales o religiosos, existen una serie de otros grupos del que "nosotros" nos diferenciamos y cuya existencia implícitamente se reconoce. Así, por ejemplo, el nacionalismo español reconoce la existencia de otras naciones como la francesa, o la identidad cristiana se opone p.ej. la identidad musulmana y quien crea en la existencia de la raza germana reconoce implícitamente la existencia de otras razas como la eslava. A esta diferenciación binaria se opone la mera existencia del judío. El judío es la figura del tercero 
que se opone a la ordenación del mundo. La figura del judío cruza las fronteras categoriales y presenta una tercera opción. "Si triunfara el tercero, la diferenciación binaria que constituye el propio grupo y el orden del mundo sería obsoleto. Una amenaza más fundamental no es imaginable." (Holz, 2005: 31). Por ello, para el nacionalsocialismo la "cuestión judía" no sólo fue un problema alemán sino un problema para todos los pueblos, y por ello Hamás no lucha sólo contra Israel sino contra el "sionismo mundial". Los judíos se convierten así, no sólo y por su supuesto poder global, sino por su mera existencia que pone en duda el orden mundial, en "enemigo del mundo" (ibid. 36). Un $60 \%$ de los europeos encuestados por Eurostat ven en Israel, un Estado con tan sólo unos pocos habitantes más que Catalunya, la principal amenaza para la paz mundial.

Esta semántica común, subyacente entre las diferentes formas del antisemitismo, explica por un lado las alianzas tan extrañas entre ideologías diversas cuando se trata de la "cuestión judía" o de Israel. Por otro lado, se ha podido mostrar también que esta semántica subyacente es capaz de conectar con diferentes ideología básicas, sean de izquierdas, del centro o de derechas, sean tradicionales o modernas (recuerde que el antimodernismo también es una ideología moderna).

\section{La sociología y el antisemitismo}

El surgimiento de la sociología y del antisemitismo moderno no sólo coincide en el tiempo, también existe un cierto paralelismo entre sus preocupaciones. "La teoría sociológica surge como respuesta liberal a fenómenos de crisis en varios momentos del siglo XIX, mientras que el antisemitismo moderno es en sí mismo un 'esperpento de teoría social' (Zerrbild einer Gesellschaftstheorie), que ofrece en sus fantasmagorías del 'judío' y ‘judificación' una explicación de las mismas deficiencias y crisis sociales." (Stoetzler, 2010: 191). En otras palabras: la joven sociología y el antisemitismo coincidieron en la identificación de los problemas sociales de la época: individualización, pérdida de comunidad, desorientación, desarraigo, etc. Por ello, los diagnósticos de la sociología clásica se prestan para ser conectados con el antisemitismo moderno: La diferenciación entre Gemeinschaft (Comunidad) y Gesellschaft (Sociedad) de Tönnies del año 1887 es quizá el ejemplo más destacado, pero también en otros autores clásicos encontramos elementos, al menos ambiguos. De modo que "La Ética Protestante" de Weber (2001) puede ser entendida como la constatación de la necesidad de domesticar al capitalismo mediante culturas nacionales. Como "enemigo" se puede identificar fácilmente el utilitarismo, el capitalismo anglo-americano y también el "capitalismo judío" y el "capitalismo 
paria". 6 También la teoría de Durkheim de la división del trabajo puede ser entendida como una defensa de una nueva solidaridad basada en el individualismo que el autor francés posiciona contra el utilitarismo y el individualismo egoísta (Stoetzler, 2010: 180), términos que para gran parte de la población de la época eran identificados con los judíos.

Para evitar malentendidos, resulta importante destacar que el problema social del antisemitismo ha sido reconocido por muchos sociólogos, algunos de ellos como Weber y Durkheim (véase p. ej. Goldberg, 2008), se pronunciaron incluso claramente contra teorías antisemitas. La tradición racional y liberal de la sociología incluso ha motivado la sospecha de ser una ciencia judía (Stoetzler, 2010: 192). No obstante, y esto es importante para el argumento sobre las dificultades de la sociología actual de captar el antisemitismo moderno, la sociología comparte algunas figuras de pensamiento con el antisemitismo.

Un problema de la sociología después de la Segunda Guerra Mundial es que resulta imposible hablar del antisemitismo moderno sin tener en consideración el Holocausto. Aquí se muestra el gran problema de la sociología: su racionalismo. La mayor parte de las teorías e investigaciones sobre antisemitismo son racionalistas. El racionalismo respecto al antisemitismo presenta no menos de cuatro problemas.

Primero la idea de "entender" en la tradición weberiana de sociología (verstehende Soziologie). Ella se basa, en última instancia, en la definición de que la tarea de la sociología es la de entender la acción social, comprendiendo como acción aquello que tiene un sentido subjetivo. Esta vertiente se encuentra con el problema de dar sentido al "sinsentido" del Holocausto. Una de las características singulares del Holocausto es justamente su disfuncionalidad. En vez de aprovechar los judíos como soldados, ingenieros o cualquier otro tipo de mano de obra, se empleaban recursos muy escasos en tiempos de guerra para organizar su eliminación. Como ejemplo de excelencia de esta confusión, que supone algún tipo de funcionalidad social, podemos encontrar el artículo temprano de Talcott Parsons "The sociology of modern Anti-Semitism" (Parsons, 1942) que interpreta el antisemitismo como simple prejuicio cuya funcionalidad es la de elevar el estatus social de los antisemitas. No obstante, como se mostró después de la Segunda Guerra Mundial, el genocidio judío no fue un medio para otros fines, sino que fue un fin en sí mismo (Mate, 2004: 162). Reyes Mate ejemplifica el sinsentido del Holocausto con la película "Shoa" de Claude Lanzmann "Cuando alguien pregunta por algo, por la razón de algo, se le responde 'hier ist kein warum'. Aquí no se dan razones porque no las hay. El hecho es lo que debe dar que pensar y no al revés: no es el pensamiento o la razón lo que lleva hasta el hecho." (Mate, 2004: 157).

La línea funcionalista del estudio del antisemitismo, también vinculada con el nombre de Gordon Allport que, en referencia a Parsons, elabora su teoría sobre

${ }^{6}$ Sobre el papel de los judíos en la Ética Protestante y el desarrollo de esta entre la primera versión de 1904/5 y la versión más conocida de 1920 véase Gosh (2006). 
prejuicios (Allport, 1954), entiende el antisemitismo como cualquier otro prejuicio. Como parte de investigaciones sobre prejuicios, el antisemitismo ha encontrado su posición en la sociología internacional (véase también Salzborn, 2010). De esto también da fe el estudio sobre antisemitismo que el Instituto de Investigación Social realiza en su exilio en EE.UU. bajo el título "Estudios de prejuicios" y cuyo texto más famoso es quizás los Estudios sobre el carácter autoritario (Adorno, 1973).

Otra consecuencia de este entendimiento, en última instancia funcionalista del antisemitismo, es su incorporación en el área de estudios sobre el genocidio. En este sentido el Holocausto parece sólo un genocidio entre otros que el mundo está sufriendo especialmente en el siglo XX. Aunque sin duda existen muchos paralelismos entre el Holocausto y otros genocidios, la diferencia radica en que todos los demás genocidios se llevaron a cabo como manera - por supuesto bárbara - de aumentar poder o asegurar recursos. El Holocausto, por el contrario, tuvo tanta prioridad en la política nazi que se arriesgó la pérdida de la guerra con tal de eliminar a los judíos.

El segundo problema del racionalismo sociológico está vinculado con la idea de la modernidad y la Ilustración como proyecto de progreso histórico, en principio poco problemático. Es este el punto común entre "orden y progreso" comptiano y la confianza en la historia del marxismo y de la sociología postmarxista: "Los sociólogos y los marxistas se consideraban herederos de la Ilustración, ambos comparten un racionalismo que limita su perspectiva de las tendencias evolutivas de la sociedad. En la sociología esto puede apreciarse en su carácter fundamentalmente afirmativo y positivista, en los marxismos en su confianza en la historia." (Claussen, 2012: 27). En esta línea, el Holocausto no podía ser entendido más que como forma de interrupción del proyecto de la modernidad, como un accidente en el camino de la Ilustración. No obstante, como advierte Zygmunt Baumann (1989: 13), sin la modernización, el Holocausto no hubiera sido posible. Sin la industrialización, la organización racional y burocrática y el desprendimiento emocional de los trabajadores podría haber propiciado pogromos antisemitas, pero no la organización industrial del exterminio. A excepción de la Teoría Crítica, que muy pronto reconoció la Dialéctica de la Ilustración (Horkheimer y Adorno, 2010b), la sociología, después del Holocausto, siguió creyendo en el proyecto del progreso humano y social. Decía Marcel Stoetzler en un estudio sobre teoría sociológica y antisemitismo: "Cuando algunos teóricos celebrados pero de hecho aislados, incluyendo a Horkheimer, Adorno y Sartre, lo sugirieron [la idea que liberales, demócratas y socialistas podrían estar implicados en el mal del Holocausto B.H.], sus propuestas fueron simplemente ignoradas o bien neutralizadas y apartadas hacia el mundo paralelo de la alta teoría social; en otras palabras: fueron considerados demasiado difíciles, brillantes o esotéricos para ser actualmente pertinentes en el trabajo diario de discursos académicos o políticos." (Stoetzler, 2010: 165). Para la mayoría de los enfoques sociológicos el Holocausto, por tanto, sólo podría ser una interrupción pero nunca un producto del proceso de la Ilustración.

Esta posición del Holocausto como accidente histórico en el camino imparable de la ilustración comparte con la interpretación del Holocausto, como "simple" 
racismo o genocidio la característica de ser capaz de cercar la barbarie. Construye una valla alrededor de la barbarie y la aleja del proyecto sociológico de la Ilustración. Lejos de percibir el Holocausto como posibilidad de la sociedad moderna (Baumann, 1989: 12) lo percibe como su contrario, como un "vestigio pre-burgués" (Claussen, 2012: 27). He aquí el tercer problema del racionalismo sociológico: el orden conceptual como objetivo de la sociología. El fin de la sociología no es sólo entender la realidad social, sino también conceptualizarla, reduciendo su complejidad y con ello ordenarla en categorías que despojan a la realidad su temible contingencia.

El cuarto y último problema relacionado con el racionalismo de la sociología tiene igualmente que ver con la suposición weberiana de un sentido subjetivo tras las acciones humanas. Detlev Claussen resume esta crítica diciendo que el antisemitismo ha sido interpretado por la sociología dominante como una ideología "y no como formas de praxis. Esta comprensión se apoya en una deformación típica de la Ilustración, que asume el modelo del aparejador: primero existe un plan y luego es aplicado a la realidad. La sociología concibe las explicaciones antisemitas de la violencia contra los judíos como si fueran entidades espirituales, y de este modo las racionaliza en lugar de reconocerlas como lo que son: justificaciones de la violencia contra los judíos en forma de palabras y acciones" (Claussen, 2012: 31). Esta crítica hace referencia a dos enfoques que el estudio del antisemitismo tiene que incluir si no quiere caer en la trampa de la prevalencia del espíritu: por un lado tiene que tomar en consideración comprensiones desde la psicología (social) y por otro seguir una perspectiva político-económica. Adorno y Horkheimer eran bastante conscientes de esta necesidad. Incluso podríamos decir que en algunos de sus textos más conocidos como la Educación después de Auschwitz (Adorno, 1998) o los ya mencionados Estudios sobre el Carácter Autoritario (Adorno, 1973) pecan de psicologismo. Este término hace referencia a la tendencia a explicar el antisemitismo sobre todo por una falta de la fuerza del yo, una falta de autonomía o una falta de "la fuerza de reflexión, de autodeterminación, de no-participar" (Adorno 1998: 80) contra el "predomino de todos los colectivos" (ibid: 81). Para hacer justicia hay que mencionar que Adorno reconoció su error y criticó más adelante "que los autores [de la Personalidad Autoritaria] habían intentado fundamentar el antisemitismo, y a partir de él también el fascismo, de modo exclusivamente subjetivo, incurriendo en el error de que este fenómeno político-económico fuera de tipo primariamente psicológico" (cit. según Claussen, 2012: 32). En sus Elementos del Antisemitismo, Horkheimer y Adorno (2010a) incluyeron esta perspectiva político-económica claramente. Identificaron como razón económica del antisemitismo "el disfraz del dominio como producción" (182) y describieron la "apariencia socialmente necesaria" de que la esfera de circulación, tradicionalmente vinculada con los judíos, fuera responsable por la explotación (ibid. 183). Más adelante criticaron la organización industrial y técnica por sus efectos psicológicos devastadores. Con esta crítica a trabajo y dominio en el capitalismo fueron capaces de crear un puente entre el enfoque político-económico y la destrucción de la fuerza del yo. 
Resumiendo este apartado sobre los problemas de la sociología para captar el antisemitismo moderno debido a su racionalismo, se puede decir que a excepción de unos pocos autores - considerados marginalizados para la investigación diaria - la sociología ha mostrado grandes problemas para encontrar una perspectiva que no cayera en los cuatro problemas mencionados. Para la sociología cualitativa el problema del antisemitismo se hace muy patente en su afán de entender. Resulta imposible entender algo que no tiene sentido. La sociología cualitativa, por su propia lógica tiende a identificar el antisemitismo como una ideología. Los estudios cualitativos sobre el antisemitismo, por tanto, siempre tienen que analizar los discursos (p.ej. Reisigl y Wodak, 2001) o estructuras semánticas (p.ej. Holz, 2001) del antisemitismo. Para la sociología cuantitativa los problemas resultan de la incapacidad de entender y, por ende, definir y menos aún de operacionalizar el antisemitismo. En el apartado 1 de este artículo se ha mostrado la discusión sobre la dificultad de encontrar una definición única de antisemitismo. En consecuencia tampoco hay unanimidad sobre las respuestas consideradas antisemitas en los cuestionarios más utilizados sobre el tema. Adicionalmente, los cuestionarios suelen tener un problema parecido a la sociología cualitativa: contemplan sólo aquellas actitudes conscientes o pre-conscientes, es decir hechas conscientes mediante la encuesta. Y también otras técnicas que intentan cuantificar el fenómeno se ven frente a la realidad histórica de Auschwitz con la incapacidad de ofrecer una descripción adecuada. La pelea sobre la cantidad exacta de los muertos, en el mejor de los casos, resulta ridículo, en el peor bárbaro en sí mismo. La respuesta atribuida a Rudolf Hö $\beta$, comandante de Auschwitz a la acusación de haber matado 3,5 millones de judíos era "Disculpen, sólo fueron 2,5 millones" y muestra toda la frialdad de los números frente a la barbarie.

La barbarie como posibilidad de la ilustración, la posibilidad de la contingencia, esto es del no-orden social, la necesidad de una perspectiva psicológica y de una perspectiva económico-política, serían consecuencias de la experiencia histórica del antisemitismo y del Holocausto. De momento se puede afirmar, con Zygmunt Bauman, que "el Holocausto puede decirnos más sobre la situación de la sociología, que la sociología en su estado actual es capaz de añadir a nuestro conocimiento del Holocausto" (Baumann, 1989: 3).

\section{España y la sociología española}

Para entender la falta de dedicación al tema del Antisemitismo en la sociología española, no basta con señalar los problemas que tiene la sociología en general con este fenómeno. Hay que relacionar el problema general con la situación sociohistórica específica de España y de la sociología española.

En España, de forma oficial hasta el final del franquismo y de forma extraoficial en algunos círculos católicos, hasta mucho después, el judío era la antítesis religiosa a la identidad española (Renyer, 2010: 40). Hay que recordar que no se abolió definitivamente el Tribunal de la Inquisición hasta 1834 y que la Iglesia católica no 
abandonó su doctrina antijudía hasta 1965. Consecuentemente los estereotipos antijudíos se pudieron grabar sin gran resistencia en la consciencia colectiva española. Los ideólogos franquistas presentaron su anti-judaísmo de forma abierta y clara, al menos hasta el final de la Segunda Guerra Mundial. Así se puede leer por ejemplo en el semanario Arriba de la Falange del 1. De agosto de 1936: "Camarada, tienes obligación de perseguir al judaísmo, a la masonería, al marxismo y al separatismo" (cit. según Baer, 2008: 94). No obstante, estamos en gran medida delante de un "antisemitismo sin judíos" (Álvarez Chillida, 2012: 214) y el judío más que una presencia real era una proyección para todo tipo de estereotipos. Dice Álvarez Chillida: "el antisemitismo español no buscaba tanto perseguir judíos como justificar ideológicamente la propia causa frente a un enemigo heterogéneo demonizado y amalgamado a partir de la secreta conspiración judeo-masónica" (ibid. 215s).

Como se ha visto, las formas modernas del antisemitismo, aunque abandonan el prejuicio religioso, se basan en las mismas estructuras de pensamiento del antijudaísmo tradicional. Rodríguez Jiménez describe el cambio paulatino del concepto proviniente del ámbito religioso del judaísmo al concepto más secular del sionismo para describir las supuestas amenazas que está recibiendo España. Cita un "Manual de urgencia sobre el sionismo en España" que afirma durante la Transición que "la Constitución aprobada para España es masónica y marxista, para que de una forma u otra pueda ser dominada por el sionismo" (cit. según Rodríguez Jiménez, 2007: 265). El paralelismo con las afirmaciones 40 años antes es más que obvio.

Después del franquismo y hasta la actualidad se puede observar en España una disputa sobre la memoria histórica. En esta disputa hay discrepancias sobre el estatus de las víctimas y sobre la forma de hacer justicia histórica. Mientras se pugna aún por reconocer a los republicanos como principales víctimas de la dictadura franquista, poco espacio parece existir para conmemorar la memoria de otras víctimas del fascismo internacional. La dedicación a cuestiones de justicia histórica vinculadas con los judíos tiene que parecer, en este contexto, una desviación de la pregunta central por el estatus de los republicanos como grupo principal de víctimas.

En esta lucha por la narración de la historia en España, la singularidad del Holocausto $^{7}$ y su destacado papel en la historia europea e incluso mundial, corren el riesgo de ser percibidos como "disculpa" al franquismo. A pesar de los vínculos entre franquismo y nacionalsocialismo y de las simpatías frente al antisemitismo (de Pozuelo, 2012), no fue el franquismo sino el nacionalsocialismo el principal culpable del Holocausto. No obstante, para el estatus de víctima del franquismo parece importante destacar la crueldad del régimen franquista y esto puede incluir tendencias a minimizar el Holocausto y describirle como sólo uno entre los muchos genocidios históricos o sólo uno entre los muchos crímenes del fascismo mundial.

Como resultado de esta lucha por la memoria histórica se puede constatar una falta de dedicación al tema del antisemitismo dentro de la izquierda española. El

${ }^{7}$ Sobre la singularidad del Holocausto véase Mate (2004). 
antisemitismo no es considerado un problema español y en todo caso se identifica el antisemitismo con el franquismo pero no con la sociedad actual. Así pues, el antisemitismo, lejos de ser entendido como "clave civilizadora" por la izquierda, se convierte en arma contra el régimen franquista; y como tal, parece no servir para entender fenómenos actuales. En este sentido, hay que entender también la cita del ex presidente Zapatero, presentada en la introducción de este artículo y que identificaba el antisemitismo en España única- y exclusivamente con el franquismo.

La falta de dedicación al legado histórico del nacionalsocialismo y del Holocausto en España se muestra también por la "banalización" del nacionalsocialismo. Por un lado la muestra de simbología nazi es considerada como "chiquillada" y raras veces tiene consecuencias políticas o legales. Y por otro lado, el nacionalsocialismo y la persecución de los judíos se convierten en sinónimo del mal. En los debates políticos de los últimos años se pueden encontrar una gran cantidad de comparaciones entre nacionalsocialismo/persecución de los judíos y ETA, el nacionalismo catalán, la política del PP y del PSOE, los llamados escraches, la eutanasia, el aborto y muchos otros temas más. En esta línea se encuentra también el "tic español" (Elvira Lindo cit. según Baer, 2011: 514) de comparar la situación en Palestina con el Holocausto.

La falta de sensibilidad por el antisemitismo y los estereotipos anti-judíos, vinculados con un cierto embrutecimiento frente a las comparaciones de nazi, tienen como consecuencia una aceptación, o al menos una falta de escandalización pública, frente a estereotipos antisemitas. Si bien es cierto que se pueden encontrar estereotipos y caricaturas antisemitas en toda Europa, pero, como decía Alejandro Baer, "a diferencia de otros países de Europa estos discursos [en España] no son marginales sino mainstream" (Baer, 2011: 515).

En la sociología española confluyen las dos tendencias, la particularidad española y la dificultad de la sociología como ciencia de captar el fenómeno del antisemitismo. Ya en su clásico "Problemas sociales", el primer catedrático de Sociología de España, Manuel Sales y Ferré quien ocupa su cátedra desde finales del siglo XIX, podemos ver el vínculo del antisemitismo "moderno", basado en las nuevas teorías raciales con el estereotipo antijudío de procedencia religiosa: "La raza semita ha realizado grandes empresas, ha fundado Estados duraderos y extensos, ha contribuido notablemente a la obra de la cultura; pero no puede negarse que, en facultades conceptivas y aptitudes sociales, es de condición inferior a los arios. Jamás el semita ha concebido el mundo sino como una unidad simple e indiferenciada, sin oposición ni variedad interior. Jehová y Alá son conceptos simples, absolutos, que no admiten, al lado ni debajo de ellos, ninguna otra hipóstasis..." (Sales y Ferré, 1910: 86). La cristalización académica de la sociología en España en comparación con el resto de Europa se demora $^{8}$ a causa del recelo con el que el franquismo observa esta ciencia. Esto significa, por un lado que en la sociología, muchas veces

${ }^{8}$ La tercera cátedra de sociología se creó 55 años después de la primera, en 1954. 
ubicada en las facultades de Derecho, dominaban "filosofía social, catolicismo social, krausismo, positivismo, funcionalismo, etc." (Sánchez Vera, 2003: 254). En otras palabras: eran fuertemente representadas aquellas vertientes con más dificultades estructurales para comprender al antisemitismo. Por otro lado, con la apertura de la sociología a posiciones abiertamente marxistas después del franquismo, la sensación social de progreso fue tan notable que resulta difícil percibir la historia y la modernidad en otros términos diferentes a los de la Ilustración. La fase de la "expansión" de la sociología de 1981 a 1991 (Lamo de Espinosa, 1998: 744) fue una fase de optimismo. Difícilmente cupieron reflexiones para percibir y advertir de la barbarie en medio del progreso.

Huelga decir que el recelo del franquismo a la sociología no era infundado. La sociología por definición es la ciencia de los problemas sociales y crítica con la sociedad existente. Consecuentemente, muchos sociólogos se entienden como parte de la izquierda. Por ello, se encuentran como hegemónicos entre los sociólogos españoles - así mi hipótesis - figuras argumentativas que rechazan el racismo antiislámico, de partes de la derecha que defiende a Israel, una identificación con movimientos anticolonialistas incluyendo la tendencia a percibir conflictos internacionales en términos de colonialismo y por ende, al menos, una cierta permisividad con posiciones antisionistas que cruzan la línea hacia el antisemitismo.

\section{Conclusiones}

Los datos y reflexiones aquí presentados no permiten verificar si es cierto que existe un "antisemitismo sociológico español", como afirma Alejandro Baer (2012: 101), hoy en día director del Center for Holocaust \& Genocide Studies en Minnesota. No obstante, no sólo se ha podido mostrar la falta de investigaciones sobre antisemitismo desde la sociología española, sino también se han presentado posibles causas ubicadas tanto en la sociología en general como en el contexto socio-histórico español. La defensa que se quiere hacer aquí de la importancia del estudio del antisemitismo en España se basa sobre todo en dos observaciones: la primera es que, España es uno de los países de la UE con índices de prejuicios antijudíos más elevados y todavía existe una alta incidencia de antisemitismo cotidiano e incluso antisemitismo delictivo. El estudio, y con ello la sensibilización social sobre el tema, puede aportar su grano de arroz para caminar hacia la desaparición del lastre del antisemitismo en todas sus vertientes. Y segundo, como hemos visto, la reflexión sobre el antisemitismo puede ayudar a la sociología a reconocer sus propios problemas epistemológicos. Puede servir para criticar y mejorar los instrumentos de investigación sociológicos, mostrar los límites del enfoque sociológico y poner al descubierto la importancia del trabajo interdisciplinar para el entendimiento de ciertos fenómenos sociales. En este sentido, el antisemitismo, lejos de ser un tema marginal, podría ser considerado tema clave en el proceso civilizador y ayudarnos a descifrar la sociedad actual. 


\section{Agradecimientos}

Quiero agradecer las observaciones críticas, la revisión desinteresada y los valiosos comentarios recibidos por parte de Estrella Israel, Vicent Flor y José Antonio Zamora.

\section{Bibliografía}

Adorno, T.W. (1998): "Educación después de Auschwitz", en Educación para la emancipación, Madrid, Morata, pp. 79-92.

Adorno, T.W. (1973): Studien zum autoritären Charakter, Frankfurt, Suhrkamp.

Allport, G. W. (1954): The Nature of Prejudice, Reading, Addison-Wesley Publishing.

Álvarez Chillida, G. (2012): "El antisemitismo en España", en Mate, R. y R. Forster (eds.) El judaísmo en Iberoamérica, Madrid, Trotta, pp. 197-220.

Anti-Defamation League (ADL) (2014): ADL Global 100. Disponible en web: http://global100.adl.org/.

Arendt, H. (2006): Sobre los orígenes del totalitarismo, Madrid, Alianza.

Baer, A. (2008): "Entre el antisemitismo y la solidaridad. La noche de los cristales rotos y la España de la guerra civil". En: Israel, E., J. Garzón, A. Baer, A. Benasuly y U. Macías: Los judíos en la España contemporánea. Apuntes históricos y jurídicos, Madrid, Hebraica Ediciones, pp. 89-104.

Baer, A. (2011): "Los vacíos de Sefarad. La memoria del Holcausto en España", Política y Sociedad, 48 (3), pp. 501-518.

Baer, A. (2012): "Memoria de Auschwitz y antisemitismo secundario... y tres tesis sobre el prejuicio anti-judío en la España actual", Constelaciones - Revista de Teoría Crítica, 4, pp. 99-118.

Baer, A. y P. López (2012): "The blind spots of secularization. A qualitative approach to the study of antisemitism in Spain", European Societies 24 (2), pp. 203-221.

Bauman, Z. (1989): Modernity and the Holocaust, New York, Cornell University Press. (Edición en castellano, Modernidad y Holocausto, Madrid, Sequitur).

Beck, U. (2001): La sociedad del riesgo: hacia una nueva modernidad, Barcelona, Paídos.

Broder, H. (1986): Der ewige Antisemit: über Sinn und Funktion eines beständigen Gefühls, Frankfurt, Fischer.

Claussen, D. (2012): "Dialéctica entre ciencia y cosmovisión. Sobre el antisemitismo en la sociología", Constelaciones - Revista de Teoría Crítica, 4, pp. 25-33.

De Pozuelo, E. M. (2012): El franquismo, cómplice del Holocausto, Madrid, Librosdevanguardia.

Emergui. S. (2009): “Zapatero, a un diario israelí: 'Antisemitismo había con Franco'”. El Mundo del 15 de octubre 2009. Disponible en web: http://www.elmundo.es/elmundo/2009/10/15/espana/1255597824.html. 
European Forum on Antisemitism (2005): Antisemitismo (Definición de trabajo). Disponible en web: http://www.european-forum-on-antisemitism.org/workingdefinition-of-antisemitism/espanol-spanish/.

Goldberg, C. A. (2008): “Introduction to Emile Durkheim's 'Anti-Semitism and Social Crisis", Sociological Theory, 26 (4), pp. 299-321.

Gosh, P. (2006): "The place of Judaism in Max Weber's Protestant Ethic", Zeitschrift für Neuere Theologiegeschichte, 12 (2), pp. 208-261.

Habermas, J. (1981): Teoría de la acción comunicativa, Madrid, Taurus, reed. Madrid, Trotta, 2009.

Herzog, B. (2011): "Exclusión Discursiva - Hacia un nuevo concepto de exclusión social", Revista Internacional de Sociología, 69 (3).

Holz, K. (2001): Nationaler Antisemitismus. Wissenssoziologie einer Weltanschauung, Hamburg, HIS.

Holz, K. (2005): Die Gegenwart des Antisemitismus. Islamistische, demokratische und antizionistische Judenfeindschaft, Hamburg, Hamburger Edition.

Horkheimer, M. y T.W. Adorno (2010a): "Elemente des Antisemitismus". En: Dialektik der Aufklärung, Frankfurt, Suhrkamp, pp. 177-217 (Edición en castellano: (2007): Elementos del antisemitismo“, en Dialéctica de la ilustración, Madrid, Akal, pp. 193-221).

Horkheimer, M. y T.W. Adorno (2010b): Dialektik der Aufklärung, Frankfurt, Suhrkamp (Edición en castellano (2007): Dialéctica de la ilustración, Madrid, Akal).

Lamo de Espinosa E. (1998): "Sociología en España", en Giner S., E. Lamo y C. Torres (eds.) Diccionario de Sociología, Madrid, Alianza, pp. 741-745.

Mate, R. (2004): "La singularidad del Holocausto", en VV.AA., En defensa de Israel, Madrid, Certeza, pp. 151 - 174.

Middle East Media Research Institute (MEMRI) (2006): The Covenant of the Islamic Resistance Movement - Hamas. Disponible en web: http://europenews.dk/files/hamas_charta_17_02_06.pdf.

Observatorio de Antisemitismo en España (2013): Informe sobre el Antisemitismo en España durante el año 2012. Disponible en web: http://observatorioantisemitismo.fcje.org/wpcontent/uploads/wpcf7_uploads//2009/10/informe-antisemitismo-2012.pdf.

Parsons, T. (1942): "The Sociology of Modern Anti-Semitism", en Graeber, I. y S.H. Brit (eds.), Jews in a Gentile World. The Problem of Anti-Semitism, New York, McMillan, pp. 101-122.

Piper, E. R. (ed.) (1987): Historikerstreit. Die Dokumentation der Kontroverse um die Einzigartigkeit der nationalsozialistischen Judenvernichtung, Múnich, Piper.

Postone, M. (1986): "Anti-Semitism and National Socialism: Notes on German Reactions to 'Holocaust"', en Rabinbach, A. y J. Zipes (eds.) Germans and Jews Since the Holocaust, New York, Holmes and Meier, pp. 96-115.

Reisigl, M. y R. Wodak (2001): Discourse and discrimination. Rhetorics of racism and anti-Semitism, London, Routledge. 
Rodríguez Jiménez, J. L. (2007): "El antisemitismo en el franquismo y en la transición", en Álvarez Chillida, G. y R. Izquierdo Benito (coords.), El antisemitismo en España, Cuenca, Ediciones de la Universidad de Castilla-La Mancha, p. 245266.

Sales y Ferré, M. (1910): Problemas Sociales, Madrid, Librería General de Victoriano Suárez.

Salzborn, S. (2010): Antisemitismus als negative Leitidee der Moderne. Sozialwissenschaftliche theorien im Vergleich, Frankfurt, Campus.

Salzborn, S. (2013): "Israelkritik oder Antisemitismus? Kriterien für eine Unterscheidung", Kirche und Israel, Neukirchener Theologische Zeitschrift, 28 (1).

Sánchez Vera, P. (2003): "Antecedentes de la Sociología en la Universidad de Murcia", Anales de derecho, 21, pp. 253-282.

Stoetzler, M. (2010): "Antisemitism, capitalism and the formation of sociological theory", Patterns of Prejudice, 44 (2), pp. 161-194.

Taguieff, P.-A. (2010): "Le sens de la batalle de Gaza", en Renyer, J., Anticatalanisme i antisionisme, avui, Barcelona, Dux, pp. 121-135.

Weber, M. (2001): La ética protestante y el espíritu del capitalismo, Madrid, Alianza.

Zamora, J. A. (2012): "Racismo, xenofobia, antisemitismo en el horizonte de los flujos migratorios. Enfoques teóricos y Teoría Crítica", ARBOR Ciencia, pensamiento y Cultura, 188 (755), pp. 591-604.

Zamora, J. A. y J. Maiso (2012): "Teoría crítica del antisemitismo", Constelaciones - Revista de Teoría Crítica, 4, pp. 133-177. 